\title{
Attraktivität der Profession Arzt stärken
}

Zentralvorstand der FMH

\begin{abstract}
Der Anteil der Ärztinnen und Ärzte, welche nicht mehr kurativ am Patienten tätig sind, liegt zwischen 8,4 und 12,9 Prozent. Das bedeutet, dass rund 80 Ärzte pro Jahr im Erwerbsalter aus der kurativen Tätigkeit aussteigen, während 800 neu ausgebildet werden. Für die grosse Mehrheit ist dieser Schritt definitiv. Gemäss der Befragung hält es lediglich etwa jeder Zehnte der Aussteiger (9\%) für eher oder sehr wahrscheinlich, wieder in den Arztberuf zurückzukehren. Die nicht mehr kurativ tätigen Ärztinnen und Ärzte geben für ihren Ausstieg am häufigsten Gründe an, die eng mit der ärztlichen Arbeitssituation zusammenhängen.
\end{abstract}

\section{Die Ausgangslage}

Die Frage nach der Anzahl Ärztinnen und Ärzte, welche nach Abschluss ihrer Ausbildung nicht mehr kurativ tätig sind, war in jüngster Vergangenheit immer wieder Thema in den Medien. So berichtete beispielsweise die NZZ am Sonntag ${ }^{1}$ über Vorschläge, wie sich die Quote an Berufsaussteigerinnen und -aussteigern reduzieren lassen könnte. Einigkeit besteht in der Haltung, dass der Ausstieg aus dem Arztberuf wenn immer möglich verhindert werden sollte. Gerade im Hinblick auf den zunehmenden Ärztemangel ist es zu vermeiden, dass Ärztinnen und Ärzte ihre kurative Tätigkeit am Patienten aufgeben.

Unbestritten ist die Tatsache, dass ärztliche Qualifikationen auch für andere Tätigkeiten wie Versicherungsmedizin, Verwaltung, Forschung etc. nützlich oder gar notwendig sind. Die Studie der FMH und des VSAO ${ }^{2}$ zeigt auf, dass der Anteil der Ärzte, welche nicht mehr kurativ am Patienten tätig sind, zwischen 8,4 und 12,9 Prozent liegt, was ca. 80 Ärzten pro Abschlussjahrgang Humanmedizin entspricht. Für die grosse Mehrheit ist der Ausstieg aus der kurativen Tätigkeit am Patienten definitiv. Gemäss der Befragung hält es nur etwa jeder Zehnte (9\%) für eher oder sehr wahrscheinlich, wieder in den Arztberuf zurückzukehren.

Die nicht mehr kurativ tätigen Ärztinnen und Ärzte geben für ihren Ausstieg am häufigsten Gründe an, die eng mit der ärztlich-kurativen Arbeitssituation zusammenhängen. Das Arbeitspensum und die Arbeitszeiten werden mit Abstand am häufigsten genannt: Gut ein
Drittel der Ärzte bezeichnen diesen Punkt als einen der drei wichtigsten Gründe für die Aufgabe ihrer kurativen Tätigkeit. Die Vereinbarkeit der ärztlichen Tätigkeit mit der Kinderbetreuung und die Arbeitsinhalte selbst werden von gut jeder fünften ausgestiegenen Person vorgebracht. Auch der nächste Faktor, das Anforderungsniveau, bezieht sich auf die Arbeit selbst. Erst danach folgen andere Gründe wie die Gesundheit, die Neuorientierung oder der Ruhestand. Weitere Faktoren begünstigen den Ausstieg aus der kurativen Tätigkeit:

- Die ausgestiegenen Ärztinnen und Ärzte haben ein kritischeres Bild des Arztberufes als ihre noch kurativ tätigen Kollegen.

- Die nicht mehr kurativ tätigen Ärzte machen die negativen Erfahrungen wissentlich, d.h. sie kennen die Herausforderungen bereits am Ende ihres Medizinstudiums.

- Eine unbefriedigende Situation im Arztberuf wird etwa gleich häufig als Grund für die Aufgabe angegeben wie attraktive Alternativen, die sich eröffneten.

\section{Die Argumente}

Um den Ausstieg aus der kurativen Tätigkeit zu vermeiden, stehen aus Sicht der FMH folgende Massnahmen im Vordergrund:

- Umsetzung der geltenden gesetzlichen Vorgaben Alle Assistenzärztinnen und -ärzte unterstehen seit dem 1. Januar 2005 dem Arbeitsgesetz. Dies ist vie- 
lerorts nach wie vor noch nicht in die Praxis umgesetzt.

- Schaffung von zeitgemässen Arbeitsbedingungen Die FMH und der VSAO setzen sich für zeitgemässe Arbeitsbedingungen ein, um die Vereinbarkeit von Familie, Freizeit und Beruf zu verbessern. Arbeitspensum, planbare und regelmässige Einsatzzeiten, Teilzeitstellen sowie die Mitsprache der Ärzte bei der Einsatzplanung sind wichtige Aspekte für die gewünschte Vereinbarkeit. In diesem Kontext muss die Schaffung von betriebsnahen Kinderbetreuungsplätzen mit genügend langen Öffnungszeiten geprüft werden.

\section{- Reduktion der administrativen Belastung} Viele Studien zeigen, dass die administrative Belastung der Ärztinnen und Ärzte sowohl im Spital als auch in der Praxis in den vergangenen Jahren laufend zugenommen hat. Die Attraktivität des Arztberufes kann gesteigert werden, indem die Zeit mit dem Patienten wieder im Mittelpunkt steht und dadurch die sinnstiftenden Arbeitsinhalte und die Wertschätzung erhöht werden.
- Sensibilisierung während der gesamten Laufbahn Ärztinnen und Ärzte sind im Beruf stark gefordert manchmal so stark, dass Körper und Seele darunter leiden und es $\mathrm{zu}$ gesundheitlichen Beschwerden kommt. Bereits die angehenden Ärzte müssen in der Ausbildung auf die verschiedenen Herausforderungen des Arztberufes sensibilisiert werden. Dieser Thematik ist während der Weiter- und Fortbildung weiterhin aktiv Raum zu geben.

\section{Unsere Forderungen}

1. Schaffung von Teilzeitstellen in allen Bereichen und Hierarchiestufen

2. Abbau von administrativer Belastung

3. Förderung von Kinderkrippen und Kinderbetreuung mit voller Abdeckung der (unregelmässigen) Arbeitszeiten

4. Einhaltung der geltenden gesetzlichen Vorgaben

5. Unterstützung bei der Praxiseröffnung und -übernahme

6. Förderung von sinnstiftenden Arbeitsinhalten und Wertschätzung

7. Gesundheitsförderung und Prävention für Ärzte 